\title{
Interpolating sequences for weighted Bergman spaces on strongly pseudoconvex bounded domains
}

\author{
Hamzeh Keshavarzi
}

\begin{abstract}
Let $0<p<\infty, \beta>-1$, and $\Omega$ be a strongly pseudoconvex bounded domain with a smooth boundary in $\mathbb{C}^{n}$. We will study the interpolation problem for weighted Bergman spaces $A_{\beta}^{p}(\Omega)$. In the case, $1 \leq p<\infty$, and $\beta>\max \{n(2 p-1)-1, n(2 q-1)-1\}$, where $q$ is the conjugate exponent of $p$ (let $q=1$, for $p=1$ ), we show that a sequence in $\mathbb{B}_{n}$, the unit ball in $\mathbb{C}^{n}$, is interpolating for $A_{\beta}^{p}\left(\mathbb{B}_{n}\right)$ if and only if it is separated.

MSC(2010): primary: 47B33, secondary: 47B38; $32 A 37$

Keywords: Strongly pseudoconvex bounded domains, interpolating sequences, weighted Bergman spaces.
\end{abstract}

\section{Introduction}

In this paper, we address the interpolating problem on the weighted Bergman spaces $A_{\beta}^{p}(\Omega)$. Let us first present some notations and a brief history:

For a positive integer $n$, let $\Omega$ be a strongly pseudoconvex bounded domain with a smooth boundary in $\mathbb{C}^{n}$. Two examples of these domains are $\mathbb{D}$ (the open unit disk in the complex plane) and $\mathbb{B}_{n}$ (the open unit ball in $\mathbb{C}^{n}$ ). See [12] for an account of strongly pseudoconvex bounded domains. Throughout the paper, $a$ is a fixed point in $\Omega$. Space $H(\Omega)$ is the class of all analytic functions on $\Omega$ and $H^{\infty}(\Omega)$ is the space of all bounded functions in $H(\Omega)$. Let $\nu$ be the Lebesque measure on $\Omega$ and $\delta(z)$ be the Euclidean distance of $z$ from the boundary of $\Omega$. If $0<p<\infty$ and $\beta>-1$, the weighted Bergman space is denoted by $A_{\beta}^{p}(\Omega)$ and defined as $A_{\beta}^{p}(\Omega)=L^{p}\left(\Omega, \delta^{\beta} d \nu\right) \cap H(\Omega)$. The classical weighted Bergman space is $A^{2}(\Omega)=A_{0}^{2}(\Omega)$. For $p \geq 1$, the weighted Bergman space $A_{\beta}^{p}(\Omega)$ is a Banach space and for $0<p<1$, it is a complete metric space. For more details of Bergman spaces see [7, 8, 18, 19].

If $\varphi$ is a holomorphic self-map of $\Omega$, then the composition operator $C_{\varphi}$ on $H(\Omega)$ induced by $\varphi$ is defined as $C_{\varphi} f=f \circ \varphi$. It is well-known that the composition operators that are induced by automorphisms on $\mathbb{B}_{n}$ are bounded on $A_{\beta}^{p}\left(\mathbb{B}_{n}\right)$. The author [11, Corollary 3.3] proved that an automorphism composition operator $C_{\varphi}$ is bounded on $A_{\beta}^{p}(\Omega)$ if and only if there exists some $C>0$ such that $(1 / C) \delta(z)<\delta(\varphi(z))<C \delta(z)$ for all $z \in \Omega$. Hence, $C_{\varphi}$ is bounded on all $A_{\beta}^{p}(\Omega)$ if and only if it is bounded on some $A_{\beta}^{p}(\Omega)$. Two good sorce for studying the composition operators are $[6,17]$.

Nevannlina [14] gave a necessary and sufficient condition for a sequence to be an interpolation sequence in $H^{\infty}(\mathbb{D})$. Carleson [4] with a simpler condition presented another char- 
acterization for these sequences in $H^{\infty}(\mathbb{D})$. Using interpolation sequences, Carleson solved the corona conjecture on $H^{\infty}(\mathbb{D})$ in his celebrated paper [5]. Berndtsson [3] gave a sufficient condition for $H^{\infty}\left(\mathbb{B}_{n}\right)$-interpolating sequences. Seip [16] addressed this problem on $A_{\beta}^{p}(\mathbb{D})$ and gave a complete characterization for it. However, this problem has not been solved on $A_{\beta}^{p}\left(\mathbb{B}_{n}\right)$. Jevtic et al. [10] gave some results on $A_{\beta}^{p}\left(\mathbb{B}_{n}\right)$.

In section 3 , we study the interpolating problem on the weighted Bergman spaces $A_{\beta}^{p}(\Omega)$. In the case $1 \leq p<\infty$, and

$$
\beta>\max \{n(2 p-1)-1, n(2 q-1)-1\},
$$

where $q$ is the conjugate exponent of $p$ (let $q=1$, when $p=1$ ), we show that separated sequences are interpolating for $A_{\beta}^{p}(\Omega)$. Also, for these $\beta$ values, we prove that a sequence in $\mathbb{B}_{n}$ is $A_{\beta}^{p}\left(\mathbb{B}_{n}\right)$-interpolating if and only if it is separation.

The notation $A \lesssim B$ on a set $S$ means that, independent of any $a \in S$, there exists some positive constant $C$ such that $A(a) \leq C B(a)$. In this case, we say that $\{A\}$ is, up to a constant, less than or equal to $\{B\}$. Also, we use the notation $A \simeq B$ on $S$ to indicate that there are some positive constants $C$ and $D$ such that $C B(a) \leq A(a) \leq D B(a)$ for each $a \in S$.

\section{Preliminaries}

The Kobayashi metric. The infinitesimal Kobayashi metric $F_{K}: \Omega \times \mathbb{C}^{n} \rightarrow[0, \infty)$ is defined as

$$
F_{K}(z, w)=\inf \left\{C>0: \exists f \in H(\mathbb{D}, \Omega) \text { with } f(0)=z, f^{\prime}(0)=\frac{w}{C}\right\} .
$$

Where $H(\mathbb{D}, \Omega)$ is the space of analytic functions from $\mathbb{D}$ to $\Omega$. Let $\gamma:[0,1] \rightarrow \Omega$ be a $C^{1}$-curve. The Kobayashi length of $\gamma$ is defined as:

$$
L_{K}(\gamma)=\int_{0}^{1} F_{K}\left(\gamma(t), \gamma^{\prime}(t)\right) d t
$$

For $z, w \in \Omega$, the Kobayashi metric function is defined as

$$
\beta(z, w)=\inf \left\{L_{K}(\gamma) ; \gamma \text { is } C^{1}-\text { curve with } \gamma(0)=z \text { and } \gamma(1)=w\right\} .
$$

The Kobayashi metric of a strongly pseudoconvex bounded domain is a complete metric (see [1, Corollary 2.3.53]).

Kobayashi balls. If $0<r<1$ and $z \in \Omega$, we shall denote by $B(z, r)$ the Kobayashi ball centered at $z$ with the radius of $r$ and define it in the following way:

$$
B(z, r)=\{w \in \Omega: \tanh \beta(z, w)<r\} .
$$

Since $\beta(.,$.$) is a complete metric, the closures of Kobayashi balls are compact. By [2, Lemmas$ 2.1 and 2.2 ], for every $0<r<1$, we have

$$
\delta(z)^{n+1} \simeq \delta(w)^{n+1} \simeq \nu(B(w, r)) \simeq \nu(B(z, r)) .
$$

for all $w \in \Omega$ and $z \in B(w, r)$.

Given $z \in \Omega, 0<r<1$, and a sequence $\left\{a_{k}\right\}$ in $\Omega$, we shall denote by $N\left(z, r,\left\{a_{k}\right\}\right)$ the number of points of $\left\{a_{k}\right\}$ contained in $B(z, r)$. 
Lemma 2.1. [2, Lemma 2.5] Let $r \in(0,1)$. Then there exists a sequence $\left\{a_{k}\right\}$ of points in $\Omega$ such that $\Omega=\bigcup_{k=0}^{\infty} B\left(a_{k}, r\right)$ and $\sup _{z \in \Omega} N\left(z, R,\left\{a_{k}\right\}\right)<\infty$, where $R=\frac{1+r}{2}$. The sequence $\left\{a_{k}\right\}$ is called an r-lattice for $\Omega$.

Also, in the proof of [2, Lemma 2.5], it has been shown that the balls $B\left(a_{k}, r / 3\right)$ are disjoint.

Lemma 2.2. [2, Corollary 2.8] Given $r \in(0,1)$, set $R=\frac{1+r}{2}$. Then there exists a $C_{r}>0$ such that

$$
\chi(z) \leq \frac{C_{r}}{\nu(B(w, r))} \int_{B(w, R)} \chi d \nu, \quad \forall w \in \Omega, \forall z \in B(w, r),
$$

for every non-negative plurisubharmonic function $\chi: \Omega \rightarrow \mathbb{R}^{+}$.

Bergman kernel. Let $K(z, w)$ be the Bergman kernel for $A^{2}(\Omega)$. Indeed, if $f \in A^{2}(\Omega)$, then

$$
f(z)=\int_{\Omega} K(z, w) f(w) d \nu(w) .
$$

Since $K(., z)=\overline{K(z, .)} \in A^{2}(\Omega)$, we have

$$
K(z, z)=\int_{\Omega}|K(w, z)|^{2} d \nu(w)=\|K(z, .)\|_{0,2}^{2}
$$

Consider $r \in(0,1)$. There is a positive constant $\delta_{r}$ which for any $z \in \Omega$ with $\delta(z)<\delta_{r}$,

$$
|K(z, w)| \simeq \frac{1}{\delta(z)^{n+1}} \gtrsim 1, \quad w \in B(z, r) .
$$

Also, for all $z \in \Omega$,

$$
|K(z, z)| \simeq \frac{1}{\delta(z)^{n+1}} \gtrsim 1
$$

For the proof of the above inequalities see [15, page 186], [9, page 1239], and [2, Lemma 3.1, Lemma 3.2 and Corollary 3.3]. By [13, Corollary 11 and Theorem 13],

$$
\int_{\Omega}|K(z, w)|^{p} \delta(w)^{\alpha} d \nu(w) \lesssim \delta(z)^{\alpha+n+1-(n+1) p}
$$

for all $z \in \Omega, \frac{n}{n+1}<p<\infty$ and $-1<\alpha<(n+1)(p-1)$.

Carleson measures. A positive Borel measure $\mu$ on $\Omega$ will be called a $\beta$-Bergman Carleson measure if there exists a positive constant $K$ such that

$$
\int_{\Omega}|f(z)|^{p} d \mu \leq K \int_{\Omega}|f(z)|^{p} \delta(z)^{\beta} d \nu(z)
$$

for all $f$ in $A_{\beta}^{p}(\Omega)$. According to the [2] and [9], $\mu$ is a $\beta$-Bergman Carleson measure if and only if for some (every) $0<r<1$, there exists some $C_{r}>0$, where $\mu(B(z, r)) \leq C_{r} \delta(z)^{\beta+n+1}$, for all $z \in \Omega$. 


\section{Interpolating sequences for $A_{\beta}^{p}(\Omega)$}

In this section, we study the interpolating problem on $A_{\beta}^{p}(\Omega)$ and give several results about it. For $p>0$ and $\beta \in \mathbb{R}$, let

$$
\ell_{\beta}^{p}=\ell_{\beta}^{p}\left(\left\{a_{k}\right\}\right):=\left\{v=\left\{v_{k}\right\} \subset \mathbb{C}:\left\{\delta\left(a_{k}\right)^{\beta} v_{k}\right\} \in \ell^{p}\right\},
$$

Also, for $v \in \ell_{\beta}^{p}$,

$$
\|v\|_{p, \beta}^{p}:=\sum_{k=1}^{\infty}\left(\delta\left(a_{k}\right)^{\beta}\left|v_{k}\right|\right)^{p} .
$$

Definition 3.1. We say that $\left\{a_{k}\right\}$ is an interpolating sequence for $A_{\beta}^{p}(\Omega)$ or an $A_{\beta}^{p}(\Omega)$ interpolating sequence, denoted by $\left\{a_{k}\right\} \in \operatorname{Int}\left(A_{\beta}^{p}(\Omega)\right)$ if for any $\left\{v_{k}\right\} \in \ell_{(n+1+\beta) / p}^{p}$, there exists $f \in A_{\beta}^{p}(\Omega)$ such that $f\left(a_{k}\right)=v_{k}$, for all $k$.

Definition 3.2. We say that a sequence $\left\{a_{k}\right\}$ is separated if there exists $\delta>0$ such that, for all $j \neq k, \beta\left(a_{k}, a_{j}\right)>\delta$.

Proposition 3.3. For $\beta>-1$ and $p>0$, if $\left\{a_{k}\right\} \in \operatorname{Int}\left(A_{\beta}^{p}(\Omega)\right)$ is separated, then there is a constant $M>0$ (which is called the interpolation constant of $\left\{a_{k}\right\}$ ) such that $\|f\|_{p, \beta} \leq$ $M\|v\|_{p,(n+1+\beta) / p}$.

Proof. If we prove that $f \mapsto\left\{f\left(a_{k}\right)\right\}$ is a bounded operator then open mapping theorem implies the desire result. By using the assumption mentioned above, for some $\delta>0$, the balls $B\left(a_{k}, \delta\right)$ are pairwise disjoint. Thus,

$$
\|f\|_{p, \beta}^{p} \geq \sum \int_{B\left(a_{k}, \delta\right)}|f(z)|^{p} \delta(z)^{\beta} d v(z) .
$$

Using the plurisubhormonicity of $|f|^{p}$, we have

$$
\|f\|_{p, \beta}^{p} \gtrsim \sum \delta\left(a_{k}\right)^{n+1+\beta}\left|f\left(a_{k}\right)\right|^{p}=\left\|\left\{f\left(a_{k}\right)\right\}\right\|_{(n+1+\beta) / p}^{p} .
$$

the proof is complete.

It is well-known that the Bergman metric on $\mathbb{B}_{n}$ and the Kobayashi metric on $\Omega$ are automorphism invariant. Hence, it is not far from mind that interpolating sequences are automorphism invariant (that is, if $\left\{a_{k}\right\}$ is an $A_{\beta}^{p}(\Omega)$-interpolating sequence, then so is $\left\{\varphi\left(a_{k}\right)\right\}$, where $\varphi$ is an automorphism).

Theorem 3.4. Let $\varphi$ be an automorphism of $\Omega$ and the composition operator induced by it be bounded on $A_{\beta}^{p}(\Omega)$. If $\left\{a_{k}\right\}$ is an $A_{\beta}^{p}(\Omega)$-interpolating sequence, then so is $\left\{\varphi\left(a_{k}\right)\right\}$.

Proof. We define

$$
T_{\varphi} f(z)=\left(K\left(\varphi^{-1}(a), z\right)\right)^{\frac{2(n+1+\beta)}{(n+1) p}} f \circ \varphi(z),
$$

and

$$
S_{\varphi} f(z)=\left(K\left(\varphi^{-1}(a), \varphi^{-1}(z)\right)\right)^{\frac{-2(n+1+\beta)}{(n+1) p}} f \circ \varphi^{-1}(z) .
$$


We show that $T_{\varphi}$ is invertible with $S_{\varphi}$ as its inverse. For any $f$ in $A_{\beta}^{p}(\Omega)$, we have

$$
\left\|T_{\varphi} f\right\|_{p, \beta}^{p}=\int_{\Omega}\left|K\left(\varphi^{-1}(a), z\right)\right|^{\frac{2(n+1+\beta)}{n+1}}|f \circ \varphi(z)|^{p} \delta(z)^{\beta} d \nu(z)
$$

By 4.1, we have

$$
\left\|T_{\varphi} f\right\|_{p, \beta}^{p}=\int_{\Omega}\left|J_{\varphi}\left(\varphi^{-1}(a)\right) J_{\varphi}(z)\right|^{2}|K(a, \varphi(z))|^{2}|f \circ \varphi(z)|^{p} \delta(z)^{\beta} d \nu(z)
$$

Since the function $K(a, z)$ has upper and lower bounds,

$$
\left\|T_{\varphi} f\right\|_{p, \beta}^{p} \simeq \int_{\Omega}\left|J_{\varphi}(z)\right|^{2}|f \circ \varphi(z)|^{p} \delta(z)^{\beta} d \nu(z)
$$

Now by 4.2 ,

$$
\left\|T_{\varphi} f\right\|_{p, \beta}^{p} \simeq \int_{\Omega}|f(z)|^{p} \delta\left(\varphi^{-1}(z)\right)^{\beta} d \nu(z)
$$

Finally, [11, Corollary 3.3] implies that

$$
\left\|T_{\varphi} f\right\|_{p, \beta}^{p} \simeq \int_{\Omega}|f(z)|^{p} \delta(z)^{\beta} d \nu(z)=\|f\|_{p, \beta}^{p}
$$

Also, we can see that $T_{\varphi} S_{\varphi}=S_{\varphi} T_{\varphi}=I$. Therefore, by the open mapping theorem, $T_{\varphi}$ is invertible. Take $v \in \ell_{(n+1+p) / p}^{p}\left(\left\{\varphi\left(a_{k}\right)\right\}\right)$. Then

$$
\sum_{k=1}^{\infty}\left|K\left(\varphi^{-1}(a), a_{k}\right)\right|^{\frac{2(n+1+\beta)}{n+1}} \delta\left(a_{k}\right)^{n+1+\beta}\left|v_{k}\right|^{p}
$$

is, up to a constant, less than or equal to

$$
\sum_{k=1}^{\infty} \delta\left(a_{k}\right)^{n+1+\beta}\left|v_{k}\right|^{p}<\infty .
$$

Thus, there is an $F$ such that $\|F\|_{\beta, p} \lesssim\|v\|$ and

$$
F\left(a_{k}\right)=K\left(\varphi^{-1}(a), a_{k}\right)^{\frac{2(n+1+\beta)}{(n+1) p}} v_{k}
$$

Then if $G=S_{\varphi} F$, we can see that $G\left(\varphi\left(a_{k}\right)\right)=v_{k}$. The proof is complete.

According to the following theorem, if we add a finite number of points to a $A_{\beta}^{p}(\Omega)$ interpolating separated sequence, then it remains $A_{\beta}^{p}(\Omega)$-interpolating.

Theorem 3.5. Let $1 \leq p<\infty$ and $\beta>-1$. The union of a $A_{\beta}^{p}(\Omega)$-interpolating separated sequence and a finite number of points is again $A_{\beta}^{p}(\Omega)$-interpolating.

Proof. It is enough to show that the union of a $A_{\beta}^{p}(\Omega)$-interpolating separated sequence and one point is $A_{\beta}^{p}(\Omega)$-interpolating. Let $\left\{a_{k}\right\}$ be an $A_{\beta}^{p}(\Omega)$-interpolating separated sequence and $b \in \Omega \backslash\left\{a_{k}\right\}$. Hence, there is some $\delta>0$ such that $\beta\left(a_{k}, b\right)>\delta$, for all $k$. 
We first claim that it is enough to find $f \in A_{\beta}^{p}(\Omega)$ with $f\left(a_{k}\right)=0$ for all $k$ and $f(b) \neq 0$. To see this, let $\left\{v_{k}\right\} \cup\left\{v_{0}\right\} \in \ell_{(n+1+\beta) / p}^{p}\left(\left\{a_{k}\right\} \cup\{b\}\right)$ and let $g \in A_{\beta}^{p}(\Omega)$ be such that $g\left(a_{k}\right)=v_{k}$. Then the function

$$
F(z)=g(z)+\frac{v_{0}-g(b)}{f(b)} f(z)
$$

belongs to $A_{\beta}^{p}(\Omega)$, and $F\left(a_{k}\right)=v_{k}$ for all $k$ and $F(b)=v_{0}$.

Suppose that all $f \in A_{\beta}^{p}(\Omega)$ with $f\left(a_{k}\right)=0$ for all $k$ have $f(b)=0$. This implies that for any $f \in A_{\beta}^{p}(\Omega)$, the value $f(b)$ is determined by the values of $f\left(a_{k}\right)$ since the difference between two functions with the same values on $\left\{a_{k}\right\}$ vanishes at $b$.

We define the functional $\Lambda: \ell^{p} \rightarrow \mathbb{C}$ by

$$
\Lambda\left(\left\{\lambda_{k}\right\}\right)=f(b)
$$

where $f \in A_{\beta}^{p}(\Omega)$ is such that $f\left(a_{k}\right)=\delta\left(a_{k}\right)^{-\frac{n+1+\beta}{p}} \lambda_{k}$ for all $k$. Because $\left\{a_{k}\right\}$ is separated, as in Proposition 3.3, one can see that $\left\{f\left(a_{k}\right) \delta\left(a_{k}\right)^{\frac{n+1+\beta}{p}}\right\} \in \ell^{p}$, for every $f \in A_{\beta}^{p}(\Omega)$. We will show that $\Lambda$ is identically 0 , which implies that $f(b)=0$, for all $f \in A_{\beta}^{p}(\Omega)$. This is a clear contradiction.

Since $f(b)$ is determined by the values $f\left(a_{k}\right)$ which are actually independent of $f, \Lambda$ is linear. It is also continuous:

$$
\Lambda\left(\left\{\lambda_{k}\right\}\right)=f(b) \lesssim\|f\|_{p, \beta} \leq M \|\left\{\delta\left(a_{k}\right)^{-\frac{n+1+\beta}{p}} \lambda_{k}\left\|_{p, \frac{n+1+\beta}{p}} \lesssim M\right\|\left\{\lambda_{k}\right\} \|_{p}\right.
$$

where $M$ denotes the interpolation constant of $\left\{a_{k}\right\}$. Thus, if $\frac{1}{p}+\frac{1}{q}=1$, then $\Lambda \in \ell^{q}$, in the sense that there exists some $\left\{c_{k}\right\} \in \ell^{q}$ such that

$$
\Lambda(\lambda)=\sum \lambda_{k} c_{k}, \quad \forall \lambda \in \ell^{p} .
$$

Consider $\delta_{j k}$ as the Kronecker symbol and the function $f_{j} \in A_{\beta}^{p}(\Omega)$ with

$$
f_{j}\left(a_{k}\right)=\delta\left(a_{k}\right)^{-\frac{n+1+\beta}{p}} \delta_{j k}
$$

Using the definition we have:

$$
\Lambda\left(v^{j}\right)=f_{j}(b)=\sum \delta_{j k} c_{k}=c_{j}
$$

Now take the functions $F_{j}(z)=\left[\left|a_{j}-b\right|^{2}-\overline{\left(a_{j}-b\right)}(z-b)\right] f_{j}(z)$. Obviously, $F_{j} \in A_{\beta}^{p}(\Omega)$ and $F_{j}\left(a_{k}\right)=0$ for all $k$. Therefore,

$$
0=F_{j}(b)=\left|a_{j}-b\right|^{2} f_{j}(b)=\left|a_{j}-b\right|^{2} c_{j},
$$

and hence $c_{j}=0$. This, shows that $\Lambda \equiv 0$.

Let $\left\{a_{k}\right\}$ be a sequence in $\Omega$. We define the values

$$
K\left(\left\{a_{k}\right\}, p, q\right)=\sup _{k \in \mathbb{N}} \sum_{j=1}^{\infty} \delta\left(a_{k}\right)^{p} \delta\left(a_{j}\right)^{q}\left|K\left(a_{k}, a_{j}\right)\right|^{\frac{p+q}{n+1}} .
$$

Based on the above values of $K\left(\left\{a_{k}\right\}, p, q\right)$, we give sufficient conditions (Theorems 3.7 and 3.8) for a sequence in $\Omega$ to be $A_{\beta}^{p}(\Omega)$-interpolating. First, we need the following lemma. The equivalence of parts (i) and (ii) below has been given in [2, Theorem 4.2]. 
Lemma 3.6. The following conditions are equivalent.

(i) $\Gamma=\left\{a_{k}\right\}$ is the union of a finite number of separated sequences.

(ii) $\sup _{z \in \Omega} N\left(z, r,\left\{a_{k}\right\}\right)<\infty$ for all (some) $r \in(0,1)$.

(iii) $K\left(\left\{a_{k}\right\}, p, q\right)<\infty$, for all $q>n$ and all $p \leq q$.

(iv) There exist $q>n, p$ such that $K\left(\left\{a_{k}\right\}, p, q\right)<\infty$.

(v) for all $p>0$ and $q>n$

$$
\sup _{z \in \Omega} \sum_{j=1}^{\infty} \delta(z)^{p} \delta\left(a_{j}\right)^{q}\left|K\left(z, a_{j}\right)\right|^{\frac{p+q}{n+1}}<\infty
$$

(vi) $\sum_{j=1}^{\infty} \delta\left(a_{j}\right)^{q} \delta_{a_{j}}$ is a $(q-n-1)$-Carleson measure for $q>n$.

Proof. (ii) $\Rightarrow(\mathrm{v})$ : Let $0<r<1$, the plurisubhormonicity of $\left|K\left(z, a_{j}\right)\right|^{\frac{p+q}{n+1}}$ and 2.1 imply that

$$
\sum_{a_{j} \in \Gamma} \delta(z)^{p} \delta\left(a_{j}\right)^{q}\left|K\left(z, a_{j}\right)\right|^{\frac{p+q}{n+1}} \lesssim \sum_{a_{j} \in \Gamma} \delta(z)^{p} \int_{B\left(a_{j}, r\right)}|K(z, w)|^{\frac{p+q}{n+1}} \delta(w)^{q-n-1} d \nu(w)
$$

Statement (ii) tells us that the above value is, up to a constant, less than or equal to

$$
\delta(z)^{p} \int_{\Omega}|K(z, w)|^{\frac{p+q}{n+1}} \delta(w)^{q-n-1} d \nu(w)
$$

Now, 2.4 gives the desire result.

$(\mathrm{v}) \Rightarrow(\mathrm{iii}) \Rightarrow(\mathrm{iv})$ are abvious.

(iv) $\Rightarrow\left(\right.$ vi) Let $\delta(z)>\delta_{r}$. Then there is some $0<s<1$ such that $B(z, r) \subset\{w: \delta(w) \geq$ $s\}$ by the compactness of $\{w: \delta(w) \geq s\}$;

$$
\frac{\mu(B(z, r))}{\delta(z)^{q}} \leq \frac{\mu(\{w: \delta(w) \geq s\})}{\delta(z)^{q}} \lesssim 1
$$

Now Let $\delta(z)<\delta_{r}$. Let $z \in B\left(a_{k}, r\right)$ for some $k$ (otherwise $\mu(B(z, r))=0$ ). Then

$$
\mu(B(z, r))=\sum_{a_{j} \in \Gamma \cap B(z, r)} \delta\left(a_{j}\right)^{q}
$$

Using the triangle inequality and the monotonicity of tanh, if, $w \in B\left(a_{k}, r\right)$ and $r^{\prime}=$ $\tanh \left(2 \tanh ^{-1}(r)\right)$, then $B(w, r) \subseteq B\left(a_{k}, r^{\prime}\right)$. Thus, we can let $\Gamma \cap B(z, r) \subset B\left(a_{k}, r^{\prime}\right)$. Therefore, by 2.2 ,

$$
\begin{aligned}
\mu(B(z, r)) & \simeq \sum_{a_{j} \in \Gamma \cap B(z, r)} \delta\left(a_{j}\right)^{q} \delta\left(a_{k}\right)^{p}\left|K\left(a_{k}, a_{j}\right)\right|^{\frac{p}{n+1}} \\
& \simeq \delta(z)^{q} \sum_{a_{j} \in \Gamma \cap B(z, r)} \delta\left(a_{j}\right)^{q} \delta\left(a_{k}\right)^{p}\left|K\left(a_{k}, a_{j}\right)\right|^{\frac{p+q}{n+1}} \lesssim \delta(z)^{q} .
\end{aligned}
$$


(vi) $\Rightarrow$ (ii) If $\delta\left(a_{k}\right) \nrightarrow 0$, then there is a subsequence $\left\{a_{n_{k}}\right\}$ of $\left\{a_{k}\right\}$ and $\delta>0$ such that $\delta\left(a_{n_{k}}\right)>\delta$, for all $k \in \mathbb{N}$. Thus, for $f \equiv 1$;

$$
\int_{\Omega}|f|^{p} d \mu=\sum \delta\left(a_{k}\right)^{q} \geq \sum \delta\left(a_{n_{k}}\right)^{q}=\infty .
$$

This contradicts $(\mathrm{v})$. Hence, $\sup _{\delta(z)>\delta_{r}} N(z, r, \Gamma)<\infty$. Now let $\delta(z)<\delta_{r}$, hence, by 2.2 ,

$$
N\left(z_{0}, r, \Gamma\right) \lesssim \sum_{z \in \Gamma \cap B\left(z_{0}, r\right)}\left|K\left(z_{0}, z\right)\right|^{2}
$$

Inequality 2.1, the plurisubhormonicity of $\left|K\left(z, a_{j}\right)\right|^{2}$, and 2.4 imply that

$$
\begin{aligned}
N\left(z_{0}, r, \Gamma\right) & \lesssim \delta\left(z_{0}\right)^{-q} \sum_{z \in \Gamma \cap B\left(z_{0}, r\right)} \delta(z)^{q}\left|K\left(z_{0}, z\right)\right|^{2} \\
& \lesssim \delta\left(z_{0}\right)^{-q} \int_{\Omega}\left|K\left(z_{0}, w\right)\right|^{2} \delta(w)^{q-n-1} d \nu(w) \\
& \lesssim \delta\left(z_{0}\right)^{-q} \delta\left(z_{0}\right)^{q-n-1+n+1-2(n+1)}=1 .
\end{aligned}
$$

Theorem 3.7. Let $\left\{a_{k}\right\}$ be a union of a finite number of separated sequences in $\Omega$. If there exists an $m>0$ such that $K\left(\left\{a_{k}\right\}, m, n+1+\beta\right)<1$, then $\left\{a_{k}\right\}$ is $A_{\beta}^{1}(\Omega)$-interpolating.

Proof. Consider the restriction map $T: A_{\beta}^{p}(\Omega) \rightarrow \ell_{\frac{n+1+\beta}{p}}^{p}$, where $T(f)=\left\{f\left(a_{k}\right)\right\}$. Hence,

$$
\|T(f)\|_{\frac{n+1+\beta}{p}}^{p}=\sum\left|f\left(a_{k}\right)\right|^{p} \delta\left(a_{k}\right)^{n+1+\beta}=\int_{\Omega}|f|^{p} d \mu .
$$

Where $\mu=\sum \delta\left(a_{k}\right)^{n+1+\beta} \delta_{a_{k}}$. Since $\left\{a_{k}\right\}$ is a finite union of separated sequences, by Lemma $4.2, \mu$ is a $\beta$-Carleson measure. Therefore, $T$ is a bounded operator. In order to show that $T$ is onto, we define, given $v=\left\{v_{k}\right\} \in \ell_{n+1+\beta}^{1}\left(\left\{a_{k}\right\}\right)$, the approximation extension

$$
E(v)(z)=\sum v_{k} \delta\left(a_{k}\right)^{n+1+\beta+m} K\left(a_{k}, z\right)^{\frac{n+1+\beta+m}{n+1}} .
$$

Using 2.4, it is straightforward that

$$
\begin{aligned}
\|E(v)\|_{1, \beta} & \leq \int_{\Omega} \delta(z)^{\beta} \sum\left|v_{k}\right| \delta\left(a_{k}\right)^{n+1+\beta+m}\left|K\left(a_{k}, z\right)\right|^{\frac{n+1+\beta+m}{n+1}} d \nu(z) \\
& =\sum\left|v_{k}\right| \delta\left(a_{k}\right)^{n+1+\beta+m} \int_{\Omega}\left|K\left(a_{k}, z\right)\right|^{\frac{n+1+\beta+m}{n+1}} \delta(z)^{\beta} d \nu(z) \\
& \lesssim \sum\left|v_{k}\right| \delta\left(a_{k}\right)^{n+1+\beta}=\|v\|_{\ell_{n+1+\beta}^{1}\left(\left\{a_{k}\right\}\right)} .
\end{aligned}
$$

Hence, $E(v)$ is in $A_{\beta}^{1}(\Omega)$. On the other hand, $T E-I$, regarded as an operator on $\ell_{n+1+\beta}^{1}\left(\left\{a_{k}\right\}\right)$, has norm strictly smaller than 1 :

$$
\begin{aligned}
\|T E(v)-v\| & =\sum \delta\left(a_{k}\right)^{n+1+\beta}\left|T E(v)_{k}-v_{k}\right| \\
& \leq \sum \delta\left(a_{k}\right)^{n+1+\beta} \sum_{j: j \neq k}\left|v_{j}\right| \delta\left(a_{j}\right)^{n+1+\beta+m}\left|K\left(a_{k}, a_{j}\right)\right|^{\frac{n+1+\beta+m}{n+1}} \\
& =\sum_{j=1}^{\infty} \delta\left(a_{j}\right)^{n+1+\beta}\left|v_{j}\right| \sum_{k: k \neq j} \delta\left(a_{j}\right)^{m} \delta\left(a_{k}\right)^{n+1+\beta}\left|K\left(a_{k}, a_{j}\right)\right|^{\frac{n+1+\beta+m}{n+1}} \\
& \leq C\|v\| .
\end{aligned}
$$


Where, $C=K\left(\left\{a_{k}\right\}, m, n+1+\beta\right)<1$. This shows that $T$ is onto and the proof is complete.

Theorem 3.8. Let $\left\{a_{k}\right\}$ be a union of a finite number of separated sequences in $\Omega, 1<p<$ $\infty$, and $q$ be its conjugate exponent. If there exist $c_{1}, c_{2}>0$ such that $c_{1} c_{2}<1$ and

$$
K\left(\left\{a_{k}\right\}, \frac{n+1+\beta}{p}, \frac{n+1+\beta}{q}\right)<c_{1}^{q}, \quad K\left(\left\{a_{k}\right\}, \frac{n+1+\beta}{q}, \frac{n+1+\beta}{p}\right)<c_{2}^{p},
$$

then $\left\{a_{k}\right\}$ is $A_{\beta}^{p}(\Omega)$-interpolating.

Proof. Given $\left\{v_{k}\right\} \in \ell_{\frac{n+1+\beta}{p}}^{p}\left(\left\{a_{k}\right\}\right)$, take the approximate extention

$$
E(v)(z)=\sum v_{k} \delta\left(a_{k}\right)^{n+1+\beta} K\left(z, a_{k}\right)^{\frac{n+1+\beta}{n+1}} .
$$

Using the duality, the reproducing kernel for $A_{\beta}^{p}(\Omega)$, and Lemma 4.2 with $\mu=\sum \delta\left(a_{k}\right)^{n+1+\beta} \delta_{a_{k}}$, one has $\|E(v)\| \leq C\|v\|$.

On the other hand, if $T$ denotes the operator on $A_{\beta}^{p}(\Omega)$ associated with $\left\{a_{k}\right\}$, we have $\|T E-I\|<1$, since

$$
\begin{aligned}
\|T E(v)-v\| & =\sum_{j} \delta\left(a_{k}\right)^{n+1+\beta}\left|T E(v)_{k}-v_{k}\right|^{p} \\
& \leq \sum_{k=1}^{\infty}\left(\sum_{j: j \neq k} \delta\left(a_{k}\right)^{\frac{n+1+\beta}{p}} \delta\left(a_{j}\right)^{\frac{n+1+\beta}{q}}\left|K\left(a_{k}, a_{j}\right)\right|^{\frac{n+1+\beta}{n+1}}\right)^{\frac{p}{q}} \\
& \times \sum_{j: j \neq k} \delta\left(a_{k}\right)^{\frac{n+1+\beta}{p}} \delta\left(a_{j}\right)^{\frac{n+1+\beta}{q}}\left|K\left(a_{k}, a_{j}\right)\right|^{\frac{n+1+\beta}{n+1}} \delta\left(a_{j}\right)^{n+1+\beta}\left|v_{j}\right|^{p} \\
& \leq c_{1}^{p} \sum_{j=1}^{\infty} \delta\left(a_{j}\right)^{n+1+\beta}\left|v_{j}\right|^{p} \sum_{k: k \neq j} \delta\left(a_{j}\right)^{\frac{n+1+\beta}{p}} \delta\left(a_{k}\right)^{\frac{n+1+\beta}{p}}\left|K\left(a_{k}, a_{j}\right)\right|^{\frac{n+1+\beta}{n+1}} \\
& \leq\left(c_{1} c_{2}\right)^{p}\|v\| .
\end{aligned}
$$

This shows that $T E$ is invertible and so $T$ is onto.

Corollary 3.9. Let $1 \leq p<\infty$, and $\left\{a_{k}\right\}$ be a union of a finite number of separated sequences in $\Omega$. Then there is a positive constant $D$ such that if $\delta\left(a_{k}\right) \leq \frac{1}{2^{k} D}$, then $\left\{a_{k}\right\}$ is an interpolating sequence for $A_{\beta}^{p}(\Omega)$.

Proof. We prove only the case $p=1$. The case $p>1$ is obtained similarly. Let $C$ be the constant that

$$
|K(z, z)| \leq C \delta(z)^{-(n+1)}, \quad \forall z \in \Omega .
$$

The following calculations show that if $D$ is such that $C^{\frac{m+n+\beta+1}{n+1}} / D<1$, then the preceding 
theorem implies that our claim holds.

$$
\begin{aligned}
K\left(\left\{a_{k}\right\}, m, n+1+\beta\right) & =\sup \sum_{j=1}^{\infty} \delta\left(a_{k}\right)^{m} \delta\left(a_{j}\right)^{n+\beta+1}\left|K\left(a_{k}, a_{j}\right)\right|^{\frac{m+n+\beta+1}{n+1}} \\
& \leq C^{\frac{m+n+\beta+1}{n+1}} \sup \sum_{j=1}^{\infty} \delta\left(a_{k}\right)^{m} \delta\left(a_{j}\right)^{n+\beta+1} \delta\left(a_{k}\right)^{-m / 2} \delta\left(a_{j}\right)^{-(n+\beta+1) / 2} \\
& =C^{\frac{m+n+\beta+1}{n+1}} \sup \sum_{j=1}^{\infty} \delta\left(a_{k}\right)^{m / 2} \delta\left(a_{j}\right)^{(n+\beta+1) / 2} \\
& \leq C^{\frac{m+n+\beta+1}{n+1}} / D \sum_{j=1}^{\infty}\left(\frac{1}{2}\right)^{j}=C^{\frac{m+n+\beta+1}{n+1}} / D
\end{aligned}
$$

Theorem 3.7 gives the result.

Theorem 3.10. Let $1 \leq p<\infty,\left\{a_{k}\right\}$ be a union of a finite number of separated sequences in $\Omega$ and

- if $p=1$, then $\beta>n-1$, and

- if $p>1$, then $\beta>\max \{n(2 p-1)-1, n(2 q-1)-1\}$, where $q$ is the conjugate exponent of $p$.

Then $\left\{a_{k}\right\}$ is an $A_{\beta}^{p}(\Omega)$-interpolation sequence. Moreover, if $\left\{a_{k}\right\}$ is a $r$-lattice sequence, then it is an $A_{\beta}^{p}(\Omega)$-interpolation sequence.

Proof. One can see that there exists some $C>1$ such that

$$
K\left(\left\{a_{k}\right\}, \frac{n+1+\beta}{q}, \frac{n+1+\beta}{p}\right) \leq C \sum \delta\left(a_{j}\right)^{(n+1+\beta) / 2 p}
$$

By 2.1 and Lemma 4.2, we have

$$
\begin{aligned}
\sum \delta\left(a_{j}\right)^{(n+1+\beta) / 2 p} & \lesssim \sum \int_{B\left(a_{j}, r\right)} \delta(z)^{(\beta+n+1) / 2 p-(n+1)} d \nu(z) \\
& \lesssim \int_{\Omega} \delta(z)^{(\beta+n+1) / 2 p-(n+1)} d \nu(z) .
\end{aligned}
$$

Since $\beta>\max \{n(2 p-1)-1, n(2 q-1)-1\}$, the above integral is finite. Thus, there is some $N_{1} \in \mathbb{N}$ such that

$$
\sum_{j=N_{1}}^{\infty} \delta\left(a_{j}\right)^{(n+1+\beta) / 2 p} \leq \frac{1}{2 C}
$$

Hence,

$$
K\left(\left\{a_{k}\right\}_{k=N_{1}}^{\infty}, \frac{n+1+\beta}{p}, \frac{n+1+\beta}{q}\right)<1 .
$$

Similarly, we can find an $N_{2}$ such that

$$
K\left(\left\{a_{k}\right\}_{k=N_{2}}^{\infty}, \frac{n+1+\beta}{q}, \frac{n+1+\beta}{p}\right)<1 .
$$

If $N=\max \left\{N_{1}, N_{2}\right\}$, Theorem 3.8 implies that $\left\{a_{k}\right\}_{k=N}^{\infty}$ is an $A_{\beta}^{p}(\Omega)$-interpolating sequence. Finally, Theorem 3.5 completes the proof. 
Jevtic et al. [10, Lemma 1.5] proved that an interpolating sequence for $A_{\beta}^{p}\left(\mathbb{B}_{n}\right)$ is separated. Above theorem says that for suitable $\beta$, separated sequences are $A_{\beta}^{p}(\Omega)$-interpolating. Thus, the folowing result is straightforward.

Corollary 3.11. Let $1 \leq p<\infty,\left\{a_{k}\right\}$ be a sequence in $\mathbb{B}_{n}$, and

- if $p=1$, then $\beta>n-1$, and

- if $p>1$, then $\beta>\max \{n(2 p-1)-1, n(2 q-1)-1\}$, where $q$ is the conjugate exponent of $p$.

Then $\left\{a_{k}\right\}$ is an $A_{\beta}^{p}\left(\mathbb{B}_{n}\right)$-interpolating sequence if and only if it is separated.

\section{Some basic results}

The following lemma is well-known in the unit ball. Indeed, the subharmonicity of the analytic functions on the unit ball implies it. However, subharmonicity does not necessarily hold on $\Omega$. Hence, we give a short and new proof for this lemma.

Lemma 4.1. Let $0<p<\infty$ and $0<R<r$. Then there is a positive constant $C$ such that for all $f \in H(\Omega)$ :

$$
|\nabla f(z)| \leq C\left(\int_{B(a, r)}|f(w)|^{p} d \nu(w)\right)^{\frac{1}{p}}, \quad z \in B(a, R) .
$$

Proof. Let $L_{z}$ be the point evaluation at $z$ in $A^{p}(B(a, r))$. Then

$$
\frac{\partial}{\partial z_{k}} f(z)=\lim _{\lambda \rightarrow 0} \frac{f\left(z+\lambda e_{k}\right)-f(z)}{\lambda}=\left\langle f, \lim _{\lambda \rightarrow 0} \frac{L_{z+\lambda e_{k}}-L_{z}}{\lambda}\right\rangle .
$$

We put

$$
\lim _{\lambda \rightarrow 0} \frac{K_{z+\lambda e_{k}}-K_{z}}{\lambda}=S_{z_{k}, z}
$$

Also, there is a positive constant $C$ such that

$$
\left\|S_{z_{k}, z}\right\| \leq C, \quad z \in B(a, R), 1 \leq k \leq n .
$$

Thus, for $1 \leq k \leq n$,

$$
\left|\frac{\partial}{\partial z_{k}} f(z)\right|=\left|\left\langle f, S_{z_{k}, z}\right\rangle\right| \leq C\left(\int_{B(a, r)}|f(w)|^{p} d \nu(w)\right)^{\frac{1}{p}} .
$$

This completes the proof.

Let $\varphi$ be an automorphism of $\Omega$. We use $J_{\varphi}(z)$ to denote the complex Jacobian of $\varphi$ at $z$. If $J_{\varphi, R}(z)$ is the real Jacobian of $\varphi$, then it is well-known that

$$
J_{\varphi, R}(z)=\left|J_{\varphi}(z)\right|^{2}
$$

and

$$
K(z, w)=J_{\varphi}(z) \overline{J_{\varphi}(w)} K(\varphi(z), \varphi(w)),
$$

for all $z, w \in \Omega$. The above equations imply a useful relationship:

$$
\int_{\Omega} f(z) d \nu(z)=\int_{\Omega} f(\varphi(z))\left|J_{\varphi}(z)\right|^{2} d \nu(z)=\int_{\Omega} f(\varphi(z)) \frac{K(z, z)}{K(\varphi(z), \varphi(z))} d \nu(z)
$$


Lemma 4.2. Let $u \in \Omega$ such that there is an automorphism $\varphi_{u}$ on $\omega$ where $\varphi_{u}(a)=u$ and $C_{\varphi_{u}}$ is bounded on $A_{\beta}^{p}(\Omega)$. Then there is a positive constant $C$ such that

$$
C^{-1} \leq\left|\frac{K(z, u)}{K(z, v)}\right| \leq C
$$

for all $z, v \in \Omega$ with $\beta(u, v) \leq R$.

Proof. By 4.1, we have

$$
\begin{aligned}
I(z) & =\left|\frac{K(z, u)}{K(z, v)}\right|=\left|\frac{J_{\varphi_{u}^{-1}}(z) \overline{J_{\varphi_{u}^{-1}}(u)} K\left(\varphi_{u}^{-1}(z), \varphi_{u}^{-1}(u)\right)}{J_{\varphi_{u}^{-1}}(z) \overline{J_{\varphi_{u}^{-1}}(v)} K\left(\varphi_{u}^{-1}(z), \varphi_{u}^{-1}(v)\right)}\right| \\
& =\frac{\left|J_{\varphi_{u}^{-1}}(u)\right|}{\left|J_{\varphi_{u}^{-1}}(v)\right|} \times \frac{\left|K\left(\varphi_{u}^{-1}(z), a\right)\right|}{\left|K\left(\varphi_{u}^{-1}(z), \varphi_{u}^{-1}(v)\right)\right|} .
\end{aligned}
$$

For each $z \in \Omega$, we have

$$
\left|K\left(\varphi_{u}^{-1}(z), a\right)\right| \leq\|K(., a)\|_{\infty}<\infty
$$

Thus, again by 4.1

$$
I(z) \lesssim \frac{\left|J_{\varphi_{u}^{-1}}(u)\right|}{\left|J_{\varphi_{u}^{-1}}(v)\right|}=\frac{\left\|K_{u}\right\|\left\|K_{\varphi_{u}^{-1}(v)}\right\|}{\left\|K_{v}\right\|\left\|K_{a}\right\|} \lesssim\left\|K_{\varphi_{u}^{-1}(v)}\right\| .
$$

Finaly, since $\varphi_{u}^{-1}(v) \in B(a, r)$, we conclude the desired result.

Lemma 4.3. Let $0<r<1$ and $\left\{a_{k}\right\}$ be an $r$-lattice for $\Omega$. Then, for each $k \geq 1$, there exists a Borel set $D_{k}$ satisfying the following conditions:

(i) $D\left(a_{k}, r / 3\right) \subset D_{k} \subset D\left(a_{k}, r\right)$, for every $k$.

(ii) $D_{k} \cap D_{j}=\emptyset$, for $k \neq j$.

(iii) $\Omega=\cup D_{k}$.

Proof. For every $k \geq 1$, let

$$
E_{k}=D\left(a_{k}, r\right)-\cup_{j \neq k} D\left(a_{j}, r / 3\right) .
$$

Then $D\left(a_{k}, r / 3\right) \subset E_{k} \subset D\left(a_{k}, r\right)$ (since $D\left(a_{k}, r / 3\right)$ are disjoint). Also, $\left\{E_{k}\right\}$ covers $\Omega$ : if $z \in D\left(a_{j}, r / 3\right)$, for some $j \neq k$, then $z \in E_{j}$; otherwise $z \in E_{k}$.

Let $D_{1}=E_{1}$ and $D_{k+1}=E_{k+1} \backslash \cup_{i=1}^{k} D_{i} .\left\{D_{k}\right\}$ is a disjoint cover of $\Omega$ : In fact, if $z \in \Omega$, then $z \in E_{k}$ for some $k$. If $k=1$, then $z \in D_{1}$. If $k>1$, then we either have $z \in D_{i}$ for some $1 \leq i \leq k$, or $z \notin \cup_{i=1}^{k-1} D_{i}$. Thus $z \in E_{k} \backslash \cup_{i=1}^{k-1} D_{i}=D_{k}$.

For each $k \geq 1$, we have $D_{k} \subset E_{k} \subset D\left(a_{k}, r\right)$. To see that each $D_{k+1}$ contains $D\left(a_{k+1}, r / 3\right)$ $\left(D_{1}=E_{1} \supset D\left(a_{1}, r / 3\right)\right)$, we fix $k \geq 1$, and fix $z \in D\left(a_{k+1}, r / 3\right) \subset E_{k+1}$. Then $z \notin E_{i}$, for any $i \neq k+1$ which implies that $z \notin D_{i}$ for any $i \neq k+1$. This shows that

$$
z \in E_{k+1}-\cup_{i=1}^{k}=D_{k+1} \text {. }
$$




\section{References}

[1] M. Abate, Iteration theory of holomorphic maps on taut manifolds (Mediterranean Press, Cosenza, 1989) http://www.dm.unipi.it/abate/libri/libriric/libriric.html.

[2] M. Abate and A. Saracco, Carleson measures and uniformly discrete sequences in strongly pseudoconvex domains, J. London Math. Soc. 83(2), 587-605 (2011).

[3] B. Berndtsson, Interpolating sequences for $H^{\infty}$ in the ball, Nederl. Akad. Wtensch. Indag. Math. 47 (1985), 1-10.

[4] L. Carleson, An Interpolation Problem for Bounded Analytic Functions, American Journal of Mathematics Vol. 80, No. 4 (Oct., 1958), pp. 921-930.

[5] L. Carleson, Interpolations by Bounded Analytic Functions and the Corona Problem, Annals of Mathematics, Second Series, Vol. 76, No. 3 (Nov., 1962), pp. 547-559.

[6] C.C. Cowen, B.D. MacCluer, Composition Operators on Spaces of Analytic Functions. CRC Press, Boca Raton, FL, 1995.

[7] Duren, P., Schuster, A., Bergman Spaces, Mathematical Surveys and Monographs, 100, American Mathematical Society, Providence, RI, 2004.

[8] H. Hedenmalm, B. Korenblum, K. Zhu, Theory of Bergman spaces, springer-verlag, New York, 2000.

[9] Zhangjian Hu, Xiaofen Lv, and Kehe Zhu, Carleson measures and balayage for Bergman spaces of strongly pseudoconvex domains, Math. Nachr. 289, No. 10, 1237-1254 (2016).

[10] M. Jevtic, X. Massaneda, P. J. Thomas, Interpolating sequences for weighted Bergman spaces of the ball, Michigan math. j. 43, 495-517 (1996).

[11] H. Keshavarzi, Power Bounded Weighted Composition Operators and Power Bounded Below Composition Operator, Collectanea Mathematica, first online.

[12] S. Krantz, Function Theory of Several Complex Variables (Wiley, New York, 1982).

[13] H. P. Li, BMO, VMO and Hankel operators on the Bergman space of strongly pseudoconvex domains, J. Funct. Anal. 106, 375-408 (1992).

[14] R. Nevanlinna, " Uber beschrankte funktionen die in gegebenen punkten vorgeschriebene werte annehmen," Annales Academie Scientiarum Fennicae, vol. 13 (1919), No. 1.

[15] R. M. Range, Holomorphic functions and integral representations in several complex variables (Springer, Berlin, 1986).

[16] K. Seip, Beurling type density theorems in the unit disk, Invent. Math. 113 (1993), 21-39.

[17] J.H. Shapiro, Composition Operator and Classical Function Theory, springer-verlag, New York, 1993. 
[18] K. Zhu, Spaces of holomorphic functions in the unit ball (Springer-verlag, New York, 2005).

[19] K. Zhu, Operator Theory in Function Space, second edition, Math. Surveys Monogr., vol. 138, American Mathematical Society, Providence, RI, 2007.

Hamzeh Keshavarzi

E-mail: Hamzehkeshavarzi67@gmail.com

Department of Mathematics, College of Sciences, Shiraz University, Shiraz, Iran 